\title{
Prescribing antipsychotic medication for adults with intellectual disability: shared responsibilities between mental health services and primary care
}

\author{
Carol Paton, ${ }^{1,2}$ (1) Ashok Roy, ${ }^{3}$ (1) Kiran Purandare, ${ }^{1,4}$ Olivia Rendora, ${ }^{2}$ (i) Thomas R. E. Barnes ${ }^{1,2}$ (1)
}

BJPsych Bulletin (2022) 46, 311-315, doi:10.1192/bjb.2021.72

\author{
Imperial College London, UK; ${ }^{2}$ Royal \\ College of Psychiatrists, UK; ${ }^{3}$ Coventry \\ and Warwickshire Partnership NHS \\ Trust, UK; ${ }^{4}$ Central and North West \\ London NHS Foundation Trust, UK \\ Correspondence to Carol Paton \\ (carol.paton@nhs.net) \\ First received 25 Feb 2021, final revision \\ 7 Apr 2021, accepted 1 Jul 2021 \\ (c) The Author(s), 2021. Published by \\ Cambridge University Press on behalf of \\ the Royal College of Psychiatrists. This is \\ an Open Access article, distributed under \\ the terms of the Creative Commons \\ Attribution-NonCommercial-ShareAlike \\ licence (http://creativecommons.org/ \\ licenses/by-nc-sa/4.0/), which permits \\ non-commercial re-use, distribution, and \\ reproduction in any medium, provided \\ the same Creative Commons licence is \\ included and the original work is properly \\ cited. The written permission of \\ Cambridge University Press must be \\ obtained for commercial re-use.
}

\begin{abstract}
Aims and method We conducted a secondary analysis of data from a Prescribing Observatory for Mental Health audit to assess the quality of requests from intellectual disability services to primary care for repeat prescriptions of antipsychotic medication.
\end{abstract}

Results Forty-six National Health Service Trusts submitted treatment data on 977 adults with intellectual disability, receiving antipsychotic medication for more than a year, for whom prescribing responsibility had been transferred to primary care. Therapeutic effects had been monitored in the past 6 months in $80 \%$ of cases with a documented communication indicating which service was responsible for this and $72 \%$ of those with no such communication. The respective proportions were $69 \%$ and $42 \%$ for side-effect monitoring, and $79 \%$ and 30\% for considering reducing/ stopping antipsychotic medication.

Clinical implications Where continuing antipsychotic medication is prescribed in primary care for people with intellectual disability, lack of guidance from secondary care regarding responsibilities for monitoring its effectiveness may be associated with inadequate review.

Keywords Intellectual disability; antipsychotics; primary care; quality; mental health teams.
Antipsychotic medication is somewhat commonly prescribed for adults with intellectual disability in the UK and internationally. ${ }^{1-3}$ Although a proportion of these prescriptions will be for the treatment of schizophrenia, such medication is also prescribed for problem behaviours in the absence of psychotic illness, such as violence, aggression or selfinjury. ${ }^{1,4-6}$ But concerns have been raised about the extent to which antipsychotic medication is prescribed in primary care for challenging behaviour in people with intellectual disability, ${ }^{1,7}$ particularly for those with no psychiatric diagnosis. The need for careful review of longer-term antipsychotic treatment, with monitoring of both the therapeutic effects and side-effects and consideration of reduction or withdrawal of the medication as appropriate, has been highlighted. ${ }^{1,6,8}$

A clinical audit of prescribing practice in $54 \mathrm{UK}$ mental health services, conducted as part of a quality improvement programme, collected information on prescribing for 5654 adults with intellectual disability. ${ }^{5}$ Although the vast majority of those prescribed antipsychotic medication for more than a year had a documented review addressing the clinical benefits of antipsychotic medication, the monitoring of side- effects was limited, with marked variation across participating services. One untested conjecture is whether the inconsistent review and monitoring of such treatment could be partly explained by a lack of clarity about accountability between mental health services and primary care. A planned re-audit as part of the quality improvement programme provided an opportunity to collect information on the communication of such monitoring responsibilities in those cases where the prescribing of antipsychotic medication had been transferred to primary care.

\section{Method}

This paper reports a secondary analysis of data from the fourth audit in the quality improvement programme conducted by the Prescribing Observatory for Mental Health $(\mathrm{POMH})$, focusing on the quality of antipsychotic prescribing in adults with intellectual disability under the care of mental health services. ${ }^{9}$ POMH had invited all 64 member healthcare organisations in the UK to participate in this quality improvement programme. The audit data were collected 


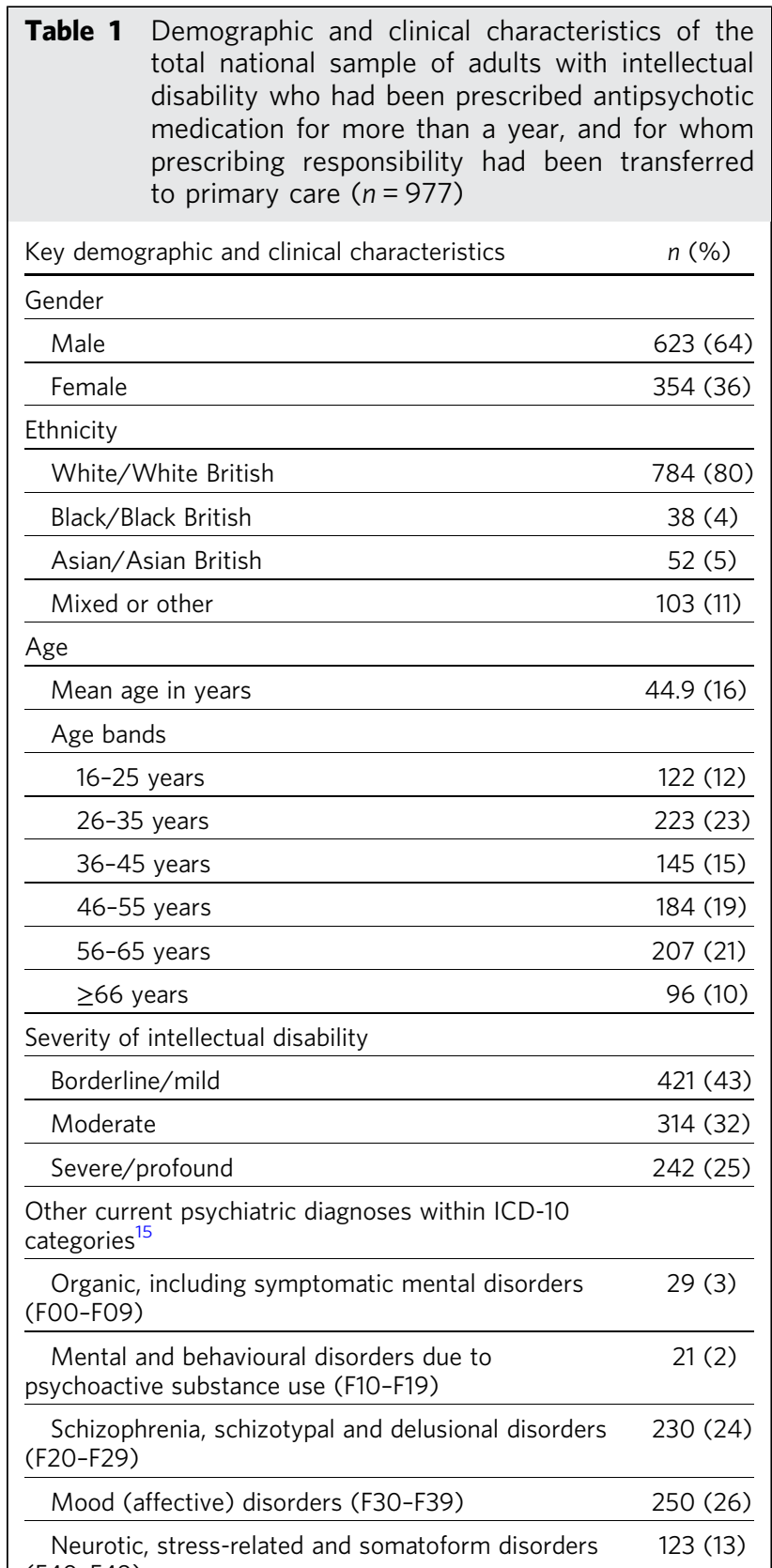
(F40-F49)

Behavioural syndrome associated with physiological 7 (1) disturbance and physical factors (F50-F59)

Disorders of adult personality and behaviour (F60-F69)

\begin{tabular}{lr}
\hline Disorders of psychological development (F80-F89) & $424(43)$ \\
\hline Behavioural and emotional disorders (F90-F98) & $104(11)$ \\
\hline Unspecified mental disorder (F99) & $1(<1)$ \\
\hline Not known & $44(5)$ \\
\hline Documented psychiatric diagnoses & $130(13)$ \\
\hline Intellectual disability (F70-F79) only & $523(54)$ \\
\hline One additional psychiatric diagnosis & $324(33)$ \\
\hline
\end{tabular}

from clinical records during February and March 2020, by clinicians and clinical audit staff, using a bespoke data collection tool. These data allowed for the assessment of clinical performance against recommended best practice, as derived from the National Institute for Health and Care Excellence (NICE) guideline for challenging behaviour in people with intellectual disability. ${ }^{4,9}$ The information collected included age, gender, ethnicity, severity of intellectual disability, comorbid psychiatric diagnoses, details of the antipsychotic medication currently prescribed and, for those patients who had received antipsychotic treatment for more than a year, the quality of clinical review addressing the effectiveness of medication (specifically, review of the therapeutic benefit and side-effects and whether there had been any consideration of dosage reduction/discontinuation of such medication). The NICE recommends that such reviews should be conducted every 6 months. ${ }^{4}$ Further information on POMH methodology is given by Barnes and Paton, ${ }^{10}$ and the methodology of this particular POMH quality improvement programme is described in more detail by Paton et al.,

In those cases where prescribing responsibility for antipsychotic medication had been transferred to primary care, information was also collected on the quality of communication between mental health services and primary care relating to which service was responsible for monitoring the therapeutic benefit and side-effects, and reviewing whether or not treatment with the medication should continue. We report here only on those cases where prescribing responsibility had been transferred to primary care, and explore whether the quality of the shared-care documentation relating to responsibilities for reviewing antipsychotic medication was associated with such reviews being documented.

\section{Data submission and analysis}

Anonymised data were submitted online during February and March 2020, using Formic software version 5.6.1 for Windows (Formic solutions, Uxbridge UK; see http://www. formic.com/survey-software/), ${ }^{11}$ and analysed with SPSS version 26 for Windows. ${ }^{12}$ Participating mental health services were sent a copy of their submitted data-set along with any data cleaning queries, allowing data entry errors to be identified and rectified.

Descriptive statistics were used for the demographic and clinical characteristics of the sample of cases for whom antipsychotic medication had been prescribed for more than a year and prescribing responsibility had been transferred from the mental health/intellectual disability team to primary care. Using $\chi^{2}$-tests, the associations were explored between the existence of clear plans relating to the transfer of prescribing responsibility and documented reviews in the past 6 months of the therapeutic effects and side-effects of antipsychotic medication, and consideration of the continuing need for such medication.

Ethical approval is not required for such audit-based quality improvement initiatives. ${ }^{13}$ POMH data are managed in accordance with its published privacy notice. ${ }^{14}$ 


\section{Results}

Forty-six healthcare organisations, predominantly National Health Service Trusts, submitted clinical data on prescribing practice for 977 adults with intellectual disability who had been prescribed antipsychotic medication for more than a year and for whom prescribing responsibility had been transferred to primary care. The demographic and clinical characteristics of this sample are shown in Table 1.

There were documented communications from mental health services to primary care indicating which service was responsible for monitoring the therapeutic effects of antipsychotic medication in $643(66 \%)$ cases, which service had responsibility for monitoring side-effects in 597 (61\%) cases, and which service was responsible for reviewing the need to continue antipsychotic medication in 223 (23\%) cases. Responsibility was allocated for all three aspects of monitoring in 178 (18\%) cases and for no aspects of monitoring in $252(26 \%)$ cases.

Where plans relating to responsibility for monitoring therapeutic effects had been communicated to primary care $(n=643)$, a review of this aspect of treatment in the past 6 months had been documented in the clinical records in 515 $(80 \%)$ cases. Where no such plan had been communicated $(n=334)$, a review of therapeutic effects had been documented in $240(72 \%)$ cases $(P=0.008)$. With respect to plans relating to responsibility for monitoring side-effects, the respective figures for documentation that such a review had taken place were 412 of the $597(69 \%)$ cases with documented plans and 158 of the $380(42 \%)$ cases without documented plans $(P<$ $0.001)$. With respect to plans relating to reviewing the need for continuing antipsychotic medication, the respective figures for documentation of such reviews were 176 of the 223 (79\%) cases with documented plans and 227 of the $754(30 \%)$ cases without documented plans $(P<0.001)$.

The majority of prescriptions of antipsychotic medication for schizophrenia spectrum disorders or affective disorders are likely to be for relapse prevention and therefore long term, so there would not be any expectation that primary care should be considering the discontinuation of such treatment in the short term. When the data on the treatment of patients with these psychiatric diagnoses were removed, 514 cases remained. In 127 (25\%) of these cases, plans for reviewing the continuing need for antipsychotic medication had been communicated to primary care, and for most $(n=111,87 \%)$ cases, there was documentation that this aspect of care had been reviewed in the past 6 months. In the 387 cases where no such plans had been communicated, such a review had been documented in just over a third $(n=136,35 \%)$ of cases.

\section{Discussion}

In our sample of adults with intellectual disability who were under the care of mental health services but were receiving their prescriptions for continuing antipsychotic medication from primary care, the quality of the clinical correspondence sent by mental health teams to primary care was associated with the quality of clinical review: when there was a clear statement about which service was responsible for reviewing the side-effects and continuing need for antipsychotic treatment, it was much more likely that such reviews had been documented in the previous 6 months.

Plans for reviewing the clinical benefits of antipsychotic medication were communicated to primary care in two-thirds of cases, and such reviews were documented in the mental health clinical records in around three-quarters of cases, with little difference between the subgroup for whom plans had been communicated and the subgroup for whom they had not. The most likely explanation for this finding is that there was an implicit understanding by both mental health services and primary care that the mental health team will be responsible for the continued monitoring of mental state and behaviour. The finding that no clinical plans relating to future reviews of the effectiveness of antipsychotic medication had been communicated to primary care for one patient in four overall is consistent with this interpretation.

Plans for monitoring the side-effects of antipsychotic medication were communicated to primary care in three out of every five cases, only a slightly lower proportion than for plans for monitoring mental state and behaviour. However, where such plans had been clearly communicated, a review of side-effects was $>50 \%$ more likely to be documented in the previous 6 months than cases where plans had not been communicated. A possible explanation for this finding is that clinicians within mental health teams considered the monitoring of side-effects and the associated physical health checks to be evidently the responsibility of primary care, as an integral part of providing continuing prescriptions for antipsychotic medication, and therefore failed to make this explicit in communications. But where a clear plan for monitoring side-effects is not communicated, primary care may assume that their role is to provide access to antipsychotic medication, with monitoring remaining the responsibility of the mental health team, as part of shared care.

Plans for reviewing the continuing need for antipsychotic medication were communicated to primary care in only a quarter of cases; for cases where such a plan had been communicated, a review of this aspect of care was more than two and a half times more likely to have been documented compared with those cases where there was no such plan. The finding was the same in the subgroup of people with a diagnosed psychotic or affective disorder, for whom continuing antipsychotic medication is likely to be indicated, as well as the subgroup prescribed antipsychotic medication for other indications (including behaviour that challenges), where the rationale for continuing treatment with antipsychotic medication may be far less convincing. This suggests that correspondence with primary care may not consistently and sufficiently address an individual's clinical circumstances and the criteria on which to base a decision to continue or withdraw antipsychotic medication, thus reinforcing any assumption by primary care that determining the need for continuing antipsychotic medication remains the remit of the mental health team. But such an assumption increases the risk that for individuals who are clinically stable and discharged to the sole care of their general practitioner, prescribing may become long-term by default, which may at least explain some of the findings from surveys of practice in primary care. ${ }^{1,6}$

Suboptimal communication across the interface between services is not a new issue nor is it specific to 
intellectual disability services or medication-related issues. ${ }^{16}$ For people prescribed antipsychotic medication for the treatment of schizophrenia, 'shared-care' agreements that clearly outline the responsibilities of primary and secondary care are commonly ratified at a systems level, but we are not aware of any such documents for the use of antipsychotic medication in people with intellectual disability who have behaviour that challenges. Although it would be possible to specify requirements for the monitoring of side-effects within such frameworks, there is insufficient evidence on which to base universal instructions for de-prescribing. ${ }^{7}$ Thus, the criteria on which to base decisions to reduce or stop antipsychotic medication in people with intellectual disability need to be individualised and clearly communicated.

In conclusion, when the responsibility for prescribing continuing antipsychotic medication for people with intellectual disability has been transferred to primary care, the monitoring and review of this medication may fall short of recommended best practice. Our findings suggest that this may partly reflect the failure of mental health services to communicate reliably with primary care about the allocation of responsibilities for reviewing antipsychotic medication. This is particularly the case for the monitoring of sideeffects and consideration of whether to reduce or discontinue the medication. The use of a formatted letter that prompts inclusion of a clear message about which service is accountable for which aspects of monitoring may be an effective quality improvement intervention.

\section{Strengths and limitations}

This study reports on findings related to the treatment of a large national sample of adults with intellectual disability, under the care of mental health services, for whom the continuing prescription of antipsychotic medication had been transferred to primary care. The findings are likely to be representative of UK practice, but should not be extrapolated to other clinical settings or patient groups.

The audit data were collected from secondary care clinical records. Thus, any clinical activity in primary care related to the monitoring and review of antipsychotic medication that had not been communicated to the intellectual disability clinical team and/or not documented in the secondary care clinical records would have been missed. Further, the audit could not determine the extent to which treatment plans included in communications from secondary care had been incorporated into the primary care records.

\section{About the authors}

Carol Paton is Joint Head of the Prescribing Observatory for Mental Health at the Centre for Quality Improvement, Royal College of Psychiatrists, UK, and an Honorary Research Fellow at the Division of Psychiatry, Imperial College London, UK. Ashok Roy is a Consultant Psychiatrist and Associate Medical Director at the Coventry and Warwickshire Partnership NHS Trust, UK. Kiran Purandare is an Honorary Clinical Senior Lecturer at Imperial College London, UK, and a Consultant in Intellectual Disability at Central and North West London
NHS Foundation Trust, UK. Olivia Rendora is a Project Officer with Prescribing Observatory for Mental Health at the Centre for Quality Improvement, Royal College of Psychiatrists, UK. Thomas R. E. Barnes is a Professor Emeritus of Clinical Psychiatry at the Division of Psychiatry, Imperial College London, UK.

\section{Data availability}

POMH data are collected for the purpose of quality improvement and belong to the individual healthcare services that collected and submitted them.

\section{Author contributions}

C.P. and T.R.E.B. designed the study. C.P., O.R. and T.R.E.B. were responsible for data analysis. All authors (C.P., A.R., K.P., O.R. and T.R.E.B.) contributed to interpreting the data, drafting the paper and revising it critically for academic content. All authors approved the final version and are accountable for its contents.

\section{Funding}

$\mathrm{POMH}$ is fully funded by subscriptions from the mental health services in the UK that participate in our quality improvement programmes.

\section{Declaration of interest}

None.

\section{References}

1 Sheehan R, Hassiotis A, Walters K, Osborn D, Strydom A, Horsfall L. Mental illness, challenging behaviour, and psychotropic drug prescribing in people with intellectual disability: UK population based cohort study. BMJ 2015; 351: h4326.

2 Bowring DL, Totsika V, Hastings RP, Toogood S, McMahon M. Prevalence of psychotropic medication use and association with challenging behaviour in adults with an intellectual disability. A total population study. J Intellect Disabil Res 2017; 61: 604-17.

3 Koch AD, Dobrindt J, Schützwohl M. Prevalence of psychotropic medication and factors associated with antipsychotic treatment in adults with intellectual disabilities: a cross-sectional, epidemiological study in Germany. J Intellect Disabil Res 2021; 65: 186-98.

4 National Institute for Health and Care Excellence (NICE). Challenging Behaviour and Learning Disabilities: Prevention and Interventions for People with Learning Disabilities Whose Behaviour Challenges. NICE Guideline [NG11]. NICE, 2015 (https://www.nice.org.uk/guidance/ng11).

5 Paton C, Bhatti S, Purandare K, Roy A, Barnes TR. Quality of prescribing of antipsychotic medication for people with intellectual disability under the care of UK mental health services: a cross-sectional audit of clinical practice. BMJ Open 2016; 6: e013116.

6 Henderson A, Mcskimming P, Kinnear D, McCowan C, Mclntosh A Allan $L$, et al. Changes over a decade in psychotropic prescribing for people with intellectual disabilities: prospective cohort study. BMJ Open 2020; 10: e036862.

7 Shankar R, Wilcock M, Oak K, McGowan P, Sheehan R. Stopping, rationalising or optimising antipsychotic drug treatment in people with intellectual disability and/or autism. Drug Ther Bull 2019; 57: 10-3.

8 Paton C, Flynn A, Shingleton-Smith A, Maclntyre S, Bhaumik S Rasmussen J, et al. Nature and quality of antipsychotic prescribing practice in UK psychiatry of learning disability services: findings of a national audit. J Intellel Disabil Res 2011; 55: 665-74. 
9 Prescribing Observatory for Mental Health. Topic 9D. Antipsychotic Prescribing in People with a Learning Disability under the Care of Mental Health Services. Prescribing Observatory for Mental Health, 2020 (Data on file).

10 Barnes TRE, Paton C. Role of the prescribing observatory for mental health. Br J Psychiatry 2012; 201: 428-9.

11 Formic Solutions. Formic Software. Formic Solutions, 2020 (http://www. formic.com/survey-software/).

12 IBM Corporation. SPSS Statistics Version 22. IBM Corporation, 2020 (https://www.ibm.com/uk-en/products/spss-statistics).

13 Health Research Authority. Do I need NHS REC review? Decision tool, 2017. Available from: http://www.hra-decisiontools.org.uk/research/docs/ DefiningResearchTable_Oct2017-1.pdf (2017, accessed February 2021).
14 Royal College of Psychiatrists. Prescribing Observatory for Mental Health (POMH-UK) Privacy Notice. Royal College of Psychiatrists, 2020 (https://www.rcpsych.ac.uk/about-us/data-protection/pomh-privacynotice).

15 World Health Organization. ICD-10: International Statistical Classification of Diseases and Related Health Problems: Tenth Revision (2nd ed). WHO, 2004.

16 Lester H. Shared care for people with mental illness: a GPs perspective. Adv Psychiat Treat 2005; 11: 133-41.

\title{
ORIGINAL PAPER
}

\section{Differences in care between younger and older patients in the 2019 English national memory service audit}

\author{
Laura D. Cook, ${ }^{1}$ Helen Souris, ${ }^{1}$ Jeremy D. Isaacs ${ }^{1,2}$ []
}

BJPsych Bulletin (2022) 46, 315-321, doi:10.1192/bjb.2021.104

${ }^{1} \mathrm{NHS}$ England/NHS Improvement (London) Dementia Clinical Network, London, UK; ${ }^{2}$ St George's University Hospitals NHS Foundation Trust,

London, UK

Correspondence to Dr Jeremy Isaacs (jeremy.isaacs@nhs.net)

First received 17 Aug 2021, final revision 17 Sep 2021, accepted 8 Oct 2021

(c) The Author(s), 2021. Published by Cambridge University Press on behalf of the Royal College of Psychiatrists. This is an Open Access article, distributed under the terms of the Creative Commons Attribution licence (https:// creativecommons.org/licenses/by/4. $0 /$ ), which permits unrestricted re-use, distribution, and reproduction in any medium, provided the original work is properly cited.
Aims and method This paper analyses how practice varied between patients aged $<65$ and $\geq 65$ years in the 2019 UK national memory service audit.

Results Data on 3959 patients were analysed. Those aged $<65$ ( $7 \%$ of the sample) were less likely than those aged $\geq 65$ to be diagnosed with dementia ( 23 v. 67\%) and more likely to receive a functional, psychiatric or no diagnosis. Younger patients were more likely to have magnetic resonance imaging; use of dementia biomarkers was low in both groups. Frontotemporal dementia and functional cognitive disorder were diagnosed infrequently. Use of dementia navigators/advisors and carer psychoeducation was similar between groups; younger patients were less likely to be offered but more likely to accept cognitive stimulation therapy.

Clinical implications Memory services seeing younger people need expertise in functional cognitive disorder, alongside clinical skills and technologies to diagnose rarer forms of dementia. Further work is needed to understand why cognitive stimulation therapy is less frequently offered to younger people.

Keywords Dementia; psychosocial interventions; clinical governance; outpatient treatment; imaging.
There are about 700000 people living with dementia in England, a figure likely to increase owing to demographic ageing. The majority of patients being assessed for, and diagnosed with, dementia, are seen in community-based memory services, usually provided by National Health Service (NHS) mental health trusts. To better understand variation between providers, and as a tool for quality improvement, the NHS London Dementia Clinical Network developed a case-note based audit of memory services which was used in London in 2015, 2016 and 2019. For the 2019 round, services throughout England were invited to participate. Initial results comparing service-level data were published by NHS
England and NHS Improvement. ${ }^{1}$ Here we present analysis of the pooled audit data-set. This provides a broad-based overview of memory service practice in England, with specific attention to how this varies by patient age.

\section{Method}

An expert reference group consisting of primary and secondary care clinicians, memory service managers and commissioners convened by the London Dementia Clinical Network developed a 'best practice' clinical data-set for a pilot audit 